\title{
relazioni
}

\section{SESSIONE 4}

\section{Le resistenze antibatteriche e antivirali}

\author{
Giovedì 10 giugno 2004, 9.00-13.00, Sala D
}

\section{S4.1}

\section{STREPTOCOCCUS PYOGENES ED ERITROMICINO-RESISTENZA}

\section{Varaldo P.E.}

\section{Istituto di Microbiologia e Scienze Biomediche. Università Politecnica delle Marche, Ancona.}

Fra le antibiotico-resistenze emergenti nei patogeni comunitari, la resistenza di S. pyogenes all'eritromicina costituisce un problema specifico e particolarmente rilevante per l'Italia, dove raggiunge incidenze fra le più elevate al mondo $(>40 \%$ in uno studio multicentrico della fine degli anni ' 90 , contro percentuali $<5 \%$ in gran parte degli altri Paesi). Incidenze di resistenza così elevate suscitano ovviamente grande preoccupazione, tanto più in un Paese come l'Italia dove, per motivi più o meno condivisibili (fra i quali l'indisponibilità della penicillina $\mathrm{V}$ ), i macrolidi sono usati molto più che altrove nel trattamento della faringite da $\mathrm{S}$. pyogenes. Inizialmente, l'emergere di un problema così rilevante finì per suscitare una serie di approcci tanto numerosi quanto non sempre ineccepibili dal punto di vista metodologico, per non parlare di iniziative legate a contrapposti interessi aziendali. Ora, a distanza di qualche anno, appare possibile ed opportuna un'obiettiva messa a punto su questo importante (soprattutto in Italia) problema di antibiotico-resistenza: tanto più che, in questi anni, diversi laboratori italiani hanno saputo cogliere l'opportunità offerta da questa situazione unica del nostro Paese per studiare e spiegare molti aspetti della eterogeneità fenotipica e genotipica, delle dinamiche epidemiologiche, dei meccanismi molecolari e delle basi genetiche di questa resistenza. Fra i risultati più significativi di questi recenti studi vale la pena di ricordare (i) la scoperta e la caratterizzazione dell'elemento genetico su cui è situato il gene mef(A), responsabile del principale sistema di efflusso dell'eritromicina; (ii) la scoperta di altri elementi genetici in cui mef(A) è associato a determinanti di resistenza ad altri antibiotici; (iii) la dimostrazione di un ruolo svolto da altri sistemi di efflusso; (iv) la documentazione di nuovi meccanismi di trasferimento e diffusione della resistenza; (v) la messa a punto di nuovi ed originali sistemi di tipizzazione; (vi) la scoperta di un'associazione fra eritromicino-resistenza e capacità di invadere cellule respiratorie, particolarmente preoccupante in quanto $\mathrm{i}$ ceppi resistenti/invasivi (in grado di sfuggire ai beta-lattamici grazie alla localizzazione intracellulare e ai macrolidi grazie alla resistenza) potrebbero essere più difficili da eradicare ed aver facilitato la grande diffusione di ceppi resistenti nel nostro Paese.

\section{S4.2}

\section{ENTEROCOCCHI E VANCOMICINA-RESISTENZA: SPECIFICITÀ ITALIANE DI UN PROBLEMA GLOBALE}

\section{Pantosti A., Caprioli A.}

\section{Istituto Superiore di Sanità, Roma}

Benchè la vancomicina sia stata messa in commercio negli anni 50, il suo uso non è stato molto frequente fino agli anni 70-80, quando la diffusione di ceppi di Staphylococcus aureus resistente alla meticillina e la necessità di trattare le coliti da antibiotici associate al Clostridium difficile, hanno richiesto l'uso massiccio di questo antibiotico. Nel giro di un decennio, sono emersi soprattutto negli ospedali degli Stati Uniti ceppi di enterococchi resistenti alla vancomicina (VRE).

I VRE sono frequenti soprattutto tra i ceppi di E.faecium, una specie spesso resistente anche ad ampicillina e aminoglicosidi. 